\title{
Therobotat the sideof the nurse: present situation and prospects
}

\author{
LauraDel Mastro ${ }^{*}$, Laura Plevani, Giacomo Cavallaro, Fabio Mosca \\ From XXI Congress of the Italian Society of Neonatology \\ Palermo, Italy. 24-26 September 2015
}

Patient safetyis a priority worldwide. According to the WHO medical errors involve 1 in 10 patients, providing damageor injury, including standing up to cause death [1]. Even the nurses can not refrain from protecting the patient as regards security, as explained in Art. 29 of the Code of ethics of nurses [2]. The complexity of the Neonatal Intensive Care Unit (NICU), the vulnerability of the neonatal population and the use of drugs "off-label" increase the risk of medical errors [3,4].

The literature reports that the risks of medical errors are related to the preparation of injectable drugs, quantified, insome cases, up to $26.90 \%$ (?) of the doses somministrate [5].

A new robotic technology, called ivSTATION ${ }^{\circledR}$ (HealthRobotics) and specifically developed for the preparation of injectable ready to administration, was introduced in our NICU to simplify the therapeutic processin the preparation stage, to optimize resources and times and especially to enhance the security. ivSTATION ${ }^{\circledR}$ is able to reconstitute powdered drugs, to obtain dilutions specifications, to verify doses prepared before making them available and to provide them with a label containing all the information required. The therapy is prepared in an aseptic environment ISO 5equipped with HEPA filters 14. The unit is equipped with UV lamps that contribute to the microbiological control overnight. ivSTATION ${ }^{\circledR}$ provides total trace ability of information about each preparation produced, making it possible to extract all the time both data specific to a single preparation and general data to be used for statistical analysis. Once the drug is prepared, it appears on the medical record through a symbol, allowing the nurse to proceed with the implementation of the successive phases of the therapeutic

\footnotetext{
* Correspondence: I.delma@libero.it

Neonatal Intensive Care Unit, Department of Clinical Sciences and

Community Health, Fondazione IRCCS Cà Granda, Ospedale Maggiore
} Policlinico, University of Milano, Italy

process, in strict observance of safety rules. The integration of the robot in the preparation of the therapies, associated with the system of the therapeutic process with the computerized system "bar code", allows a considerable decrease of the risk in both the preparation phase of identification of the patient, which are known to those where the errors are hardly detectable [6,7].

This technology is a useful tool for limiting the error and the niatrogenic clinical risk associated with medical therapy. The nurse, in charge of patients, has the opportunity, with the use of this technology, to spend more timenursing careproper.

Published: 24 September 2015

\section{References}

World Health Organization: WHO launches 'Nine patient safety solutions. 2007 [http://www.who.int/mediacentre/news/releases/2007/pr22/en/index. html].

2. 2009.

3. Samra HA, McGrath JM, Rollins W: Patient Safety in the NICU: a comprehensive review. J Perinat Neonat Nurs 2011, 25(2):123-132.

4. Frattarelli DA, Galinkin JL, Green TP, Johnson TD, Neville KA, Paul IM, Van Den Anker JN, American Academy of Pediatrics Committee on Drugs: OffLabel Use of Drugs in Children. Pediatrics 2014, 133(3):563-567.

5. Schneider MP, Cotting J, Pannatier A: Evaluation of nurses errors associated in the preparation and administration of medication in a pediatric intensive care unit. Pharm World Sci 1998, 20(4):178-182.

6. Pacheco Ramos Mde L, Arenaza Peña AE, Santiago Pérez A, Bilbao GómezMartino C, Zamora Barrios MD, Arias Fernández ML: Implementation of a robot for the preparation of antineoplastic drugs in the Pharmacy Service. Farm Hosp 2015, 39(3):137-146.

7. Poon EG, Keohane CA, Yoon CS, Ditmore M, Bane A, Levtzion-Korach O, Moniz T, Rothschild JM, Kachalia AB, Hayes J, Churchill WW, Lipsitz S, Whittemore AD, Bates DW, Gandhi TK: Effect of bar-codetechnology on the safety of medication administration. N Engl J Med 2010, 362:1698-1707. 